apparent magnitude of the supernova, about 15 , did not change appreciably between July 25 and July 26. It was not seen on a photograph (limiting magnitude 17.5) taken on July 10.

California Institute of Technology

Pasadena, California

July 1940

\title{
A NOTE ON STARS WITH NEBULOUS ABSORPTION LINES
}

\section{By P. Swings and O. Struve}

From an examination of the photographic region of the spectra of several hundred Bn stars taken at the Yerkes Observatory, Struve and $\mathrm{Wurm}^{1}$ concluded that only a very small percentage of the objects exhibit sharp lines in addition to the nebulous lines. These sharp lines are due to a surrounding gaseous shell, and the effect of the dilution of the stellar radiation consists essentially in the enhancement of those absorption lines whose lower levels are metastable. Except for this small group of peculiar spectra, the dish-shaped rotational contours were observed in all lines of all elements.

A recent paper by E. Cherrington, Jr., ${ }^{2}$ seems to indicate that the simultaneous appearance of nebulous and sharp lines is rather usual in Bn stars. From an examination of the visual region of the spectra of $18 \mathrm{Bn}$ stars, Cherrington suggests that 13 (or 72 per cent) exhibit both nebulous and sharp lines. He calls these 13 objects "super-shell stars." The spectrograms used by him were taken with the Yerkes autocollimating spectrograph attached to the 69-inch Perkins reflector.

Cherrington attributes the sharp absorption lines to $F e$ II, $N$ II, $A l$ II, $C$ II, $S$ II, $S i$ II, $C r$ II, and $T i$ II ; he does not give the wave lengths or series identifications. This result seems very strange. In the region of wave lengths greater than $\lambda 4800$, all strong lines of three elements identified by Cherrington ( $F e$ II, $C r$ II, $T i$ II $)$ start from lower metastable levels, whereas for the five other elements ( $N$ II, $A l$ II, $C$ II, $S$ II, $S i$ II), no

\footnotetext{
1 Ap. J., 88, 84, 1938.

${ }^{2}$ Pub. A.S.P., 52, 116, 1940.
} 
metastable levels are involved. Hence, the simultaneous appearance of sharp lines due to all of these elements is very improbable. On the other hand, there does not seem to be any special reason why the visual region would be favored, compared with the ordinary photographic wave-length range which contains strong lines of most of these elements.

In order to investigate this problem we have obtained, at the coudé focus of the McDonald 82-inch reflector, a highdispersion prism spectrogram of one of Cherrington's stars, $\eta$ Ursae Majoris. This spectrogram extends from about $\lambda 4380$ to $\lambda 6700$, the dispersion being $4.5 \mathrm{~A} / \mathrm{mm}$ at $\lambda 5000$. The atmospheric lines of water vapor and oxygen are fairly strong and sharp. But a systematic examination of all strong lines of the eight elements indicated by Cherrington has given a negative result. A direct comparison of the spectrogram of $\eta$ Ursae Majoris with a coudé spectrogram of $\alpha$ Cygni gives no coincidences between the sharp lines of the latter with any identifiable features of the former. The coudé spectrograms used here are of better quality than the grating spectrograms taken at the Yerkes or McDonald Observatories with the autocollimating grating spectrograph used by Cherrington. Recent spectrograms taken at the Yerkes Observatory by Greenstein and Page with the grating instrument used by Cherrington confirm our examination of the coudé spectrogram. ${ }^{3}$

We believe that there is no reason to modify our ideas concerning stellar spectra with rotationally broadened lines. The presence of an outer shell exhibiting sharp absorption lines is an exceptional feature, particularly well shown in $\zeta$ Tauri and $\varphi$ Persei.

McDonald Observatory

Fort Davis, Texas

August 22, 1940 .

3 Annual Report of the Yerkes and McDonald Obstrvatories, 1939-40. Publications of the American Astronomical Society. 